\title{
Arabidopsis CALMODULIN-BINDING PROTEIN 60b plays dual roles in plant immunity
}

Weijie Huang ${ }^{1}$, Zhongshou $\mathrm{Wu}^{1,2}$, Hainan $\operatorname{Tian}^{1}, \mathrm{Xin} \mathrm{Li}^{1,2}$ and Yuelin Zhang, ${ }^{1, *}$

${ }^{1}$ Department of Botany, University of British Columbia, Vancouver, BC V6T 1Z4, Canada

${ }^{2}$ Michael Smith Laboratories, University of British Columbia, Vancouver, BC V6T 1Z4, Canada

*For correspondence: yuelin.zhang@ubc.ca

Running title: CBP60b in plant immunity

Key words: Arabidopsis; Plant immunity; CBP60b; CBP60g; SARD1; Salicylic acid, transcription factors

\section{Significance statement:}

Arabidopsis SARD1 serves as a master transcription factor in plant immunity. In this study, we showed that CBP60b positively regulates SARD1 expression, and TIR signaling is activated when CBP60b is inactivated. 


\begin{abstract}
Arabidopsis SYSTEMIC ACQUIRED RESISTANCE DEFICIENT 1 (SARD1) and CALMODULIN-BINDING PROTEIN 60g (CBP60g) are two master transcription factors that regulate many defense-related genes in plant immunity. They are required for immunity downstream of the receptor-like protein SUPPRESSOR OF NPR1-1, CONSTITUTIVE 2 (SNC2). Constitutive defense responses in the gain-of-function autoimmune snc2-1D mutant are modestly affected by either sardl or cbp60g single mutants, but completely suppressed by the sard1 cbp60g double mutant. Here we report that CBP60b, another member of the CBP60 family, also functions as a positive regulator of SNC2-mediated immunity. Loss-of-function mutations of $C B P 60 b$ suppress the constitutive expression of SARD1 and enhanced disease

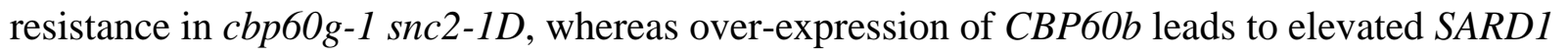
expression and constitutive defense responses. In addition, transient expression of $C B P 60 \mathrm{~b}$ in Nicotiana benthamiana activates the expression of the pSARD1::luciferase reporter gene. Chromatin immunoprecipitation assay further showed that CBP60b is recruited to the promoter region of $S A R D 1$, suggesting that it directly regulates $S A R D 1$ expression. Interestingly, knocking out $C B P 60 b$ in the wild type background leads to ENHANCED DISEASE SUSCEPTIBILITY 1 (EDS1)-dependent autoimmunity, suggesting that $\mathrm{CBP} 60 \mathrm{~b}$ is required for the expression of a guardee/decoy or a negative regulator in immunity mediated by receptors carrying an $\mathrm{N}$-terminal TIR (Toll-interleukin-1 receptor-like) domain.
\end{abstract}




\section{Introduction}

Plants have evolved sophisticated innate immune systems to fight against various pathogens (Jones and Dangl 2006, Zhou and Zhang, 2020). Upon infection, plasma membrane-localized pattern recognition receptors (PRRs) recognize pathogen/microbe-associated molecular patterns (PAMPs or MAMPs), which are conserved molecules associated with groups of pathogens, to initiate pattern-triggered immunity (PTI)(Monaghan and Zipfel, 2012). To facilitate colonization, pathogens have evolved various effector proteins to interfere with PTI (Dou and Zhou, 2012). Meanwhile, plants evolved resistance $(\mathrm{R})$ proteins, mostly in the nucleotide-binding leucine-rich repeat (NLR) protein family, to perceive the pathogen effectors and activate effector-triggered immunity (ETI)(van Wersch et al., 2020). Activation of PTI and ETI at local infection sites further leads to development of systemic acquired resistance (SAR) in the distal parts of the plants (Sun and Zhang, 2021).

One class of plant PRRs belong to the receptor-like protein (RLP) family. RLPs usually have a short cytoplasmic tail, a single transmembrane motif and an extracellular leucine-rich repeats (LRR) domain involved in binding to ligands. One of the Arabidopsis RLPs, SNC2 (SUPPRESSOR OF NPR1-1, CONSTITUTIVE 2), contributes to defense against bacteria (Zhang et al., 2010b). On one hand, loss of SNC2 results in enhanced susceptibility to bacterial pathogen Pseudomonas syringae pv tomato (Pto) DC3000. On the other hand, a gain-of-function mutation in $s n c 2-1 D$ results in constitutive Pathogenesis-Related $(P R)$ gene expression, elevated levels of the plant defense hormone SA, and enhanced resistance against the oomycete pathogen Hyaloperonospora arabidopsidis (Hpa) Noco2 (Zhang et al., 2010b). Due to constitutively activated immunity, snc2-1D plants exhibit a dwarf morphology.

\section{SAR DEFICIENT 1 (SARD1) and CALMODULIN BINDING PROTEIN 60g (CBP60g) are} two master transcription factors in plant immunity (Sun et al., 2015). Induction of SA biosynthetic genes such as ISOCHORISMATE SYNTHASE 1 (ICS1), ENHANCED DISEASE SUSCEPTIBILITY 5 (EDS5) and AVRPPHB SUSCEPTIBLE 3 (PBS3) during pathogen infection is coordinately regulated by SARD1 and CBP60g (Zhang et al., 2010a, Wang et al., 2011, Sun et $a l ., 2015)$. Pathogen-induced SA accumulation is almost completely blocked in sardl cbp60g double mutant plants (Zhang et al., 2010a, Wang et al., 2011). In addition, SARD1 and CBP60g 
are also required for activation of the biosynthesis of N-hydroxy-pipecolic acid (NHP), a signaling molecule important for both local resistance and SAR (Sun et al., 2020). They coordinately regulate pathogen-induced expression of NHP biosynthesis genes such as AGD2like defense response protein 1 (ALD1), SAR deficient 4 (SARD4) and Flavin-dependent monooxygenase 1 (FMO1) (Sun et al., 2018, Sun et al., 2020). Both SARD1 and CBP60g belong to a small protein family with eight members in Arabidopsis. Unlike CBP60g and other members in the family which have calmodulin (CaM)-binding activity (Wang et al., 2009) (Reddy et al., 2002), SARD1 does not bind CaM and is primarily regulated at transcriptional level, as overexpression of $S A R D 1$ is sufficient to activate downstream defense gene expression (Zhang $e t$ al., 2010a). Another member of the family, CBP60a, was shown to function as a negative regulator of plant immunity (Truman et al., 2013).

Both SARD1 and CBP60g contribute to autoimmunity of snc2-1D (Sun et al., 2015). Although the up-regulation of ICSI and accumulation of SA in snc2-1D are only slightly reduced in cbp60g-1 snc2-1D and sard1-1 snc2-1D double mutants, they are largely blocked in the cbp60g1 sard1-1 snc2-1D triple mutant. The sizes of cbp60g-1 snc2-1D and sard1-1 snc2-1D double mutants are slight larger than snc2-1D, whereas the cbp60g-1 sard1-1 snc2-1D triple mutant exhibits wild type morphology. These findings suggest that there are two parallel pathways downstream of SNC2, one depending on SARD1 and the other requiring CBP60g (Sun et al., 2015). How the plasma membrane localized SNC2 transduces signals to SARD1 or CBP60g is largely unknown.

To identify components involved in the SARD1-dependent defense signaling downstream of SNC2, we performed a suppressor screen in the $c b p 60 g-1 s n c 2-1 D$ background. Here we report the identification and characterization of one of the suppressors, bda7-1 (bian da 7; bian da means "becoming big" in Chinese). Positional cloning revealed that BDA7 encodes another member of the CBP60 family, CBP60b, which positively regulates the expression of SARD1.

\section{Results}

\section{Identification and characterization of bda7-1 cbp60g-1 snc2-1D}


To identify regulators of SARD1-dependent defense signaling in SNC2-mediated immunity, we mutagenized $\operatorname{cbp} 60 g-1$ snc2-1D and searched for mutants suppressing its dwarf morphology. bda7-1 was one of the mutants identified. As shown in Figure 1A, bda7-1 cbp60g-1 snc2-1D has an intermediate size compared with wild type Col-0 and cbp60g-1 snc2-1D. Quantitative RTPCR analysis showed that the elevated expression of PRI and ICS1 in cbp60g-1 snc2-1D is fully blocked by bda7-1 (Figure 1B and 1C). Consistent with the ICS1 expression, both free (Figure 1D) and total SA (Figure 1E) levels in bda7-1 cbp60g-1 snc2-1D are considerably lower than in cbp60g-1 snc2-1D. In addition, the enhanced resistance against Hpa Noco2 observed in $c b p 60 g$ 1 snc2-1D is lost in bda7-1 cbp60g-1 snc2-1D (Figure 1F). Together, these data indicate that $b d a 7-1$ suppresses the autoimmunity of $\operatorname{cbp} 60 \mathrm{~g}-1$ snc2-1D.

\section{Map-based cloning of $B D A 7$}

When bda7-1 cbp60g-1 snc2-1D was backcrossed with cbp60g-1 snc2-1D, F1 plants showed similar morphology as cbp60g-1 snc2-1D (Figure S1), indicating that $b d a 7-1$ is recessive. To map bda7-1, bda7-1 cbp60g-1 snc2-1D (in Col-0 background) was crossed with Landsberg erecta (Ler). In the F2 population, plants homozygous for both cbp60g-1 and snc2-1D with dwarf morphology were selected for linkage analysis. bda7-1 was initially mapped to a region between SSLP markers MBG8 and MUB3 on chromosome 5, and subsequently fine-mapped to a $\sim 0.8 \mathrm{Mb}$ region between MUA2 and MMN10 (Figure S2).

To identify BDA7, DNA from $50 \mathrm{~F} 2$ plants with similar size as bda7-1 cbp60g-1 snc2-1D obtained from a cross between $b d a 7-1$ cbp60g-1 snc2-1D and $\operatorname{cbp} 60 g-1$ snc2-1D were pooled and sequenced by Illumina sequencing. A single candidate gene within the $\sim 0.8 \mathrm{Mb}$ mapped region, AT5G57580, was found, which carries a homozygous $\mathrm{C}$ to $\mathrm{T}$ mutation in its coding sequence (Figure 2A and Figure S3), resulting in the substitution of Leu148 with Phe (Figure 2A).

\section{Loss of $C B P 60 b$ suppresses the autoimmunity of $\operatorname{cbp} 60 \mathrm{~g}-1 \mathrm{snc} 2-1 \mathrm{D}$}

To test whether AT5G57580 is indeed required for the autoimmunity of $c b p 60 g-1$ snc2-1D,

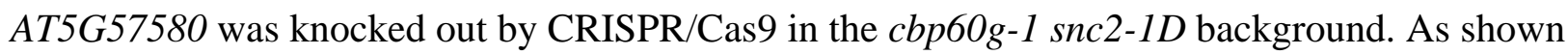
in Figure 2B, the newly generated AT5G57580 CRISPR alleles suppressed cbp60g-1 snc2-1D 
similarly as $b d a 7-1$. Sanger sequencing confirmed that these lines carry homozygous deletion mutations in BDA7 (Figure S4). Similar to $b d a 7-1$, these deletion mutants also suppress the constitutive expression of PRI and ICS1 (Figure 2C and 2D), elevated SA levels (Figure S5) and enhanced resistance against $\mathrm{Hpa}$ Noco2 in cbp60g-1 snc2-1D (Figure 2E), suggesting that $A T 5 G 57580$ is required for the constitutively immune responses in $\operatorname{cbp} 60 \mathrm{~g}-1$ snc2-1D.

To further confirm that suppression of the cbp60g-1 snc2-1D mutant phenotype in bda7-1 cbp60g-1 snc2-1D is caused by the mutation in AT5G57580, we transformed a construct expressing wild-type $A T 5 G 57580$ with a C-terminal $3 \times \mathrm{HA}$ tag under the control of its native promoter into bda7-1 cbp60g-1 snc2-1D. As shown in Figure S6, the AT5G57580-3HA transgenic lines have similar morphology as cbp60g- 1 snc2-1D, indicating that $A T 5 G 57580-3 H A$ can complement the bda7-1 mutation. Since AT5G57580 encodes CBP60b, we renamed bda7-1 as $c b p 60 b-1$.

The Leu148 residue in CBP60b is highly conserved among all CBP60 family members, except for CBP60a (Figure S7A), which functions as a negative regulator of plant immunity (Truman et

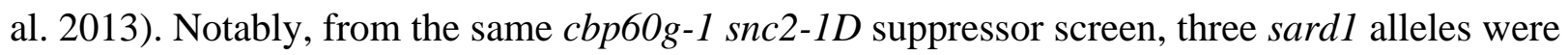
found to carry mutations affecting Gly143, a residue close to the conserved Leu residue (Figure S7B). These data suggest that this region is important for the functions of CBP60 family proteins.

\section{CBP60b regulates $S A R D 1$ expression}

As the elevated ICS1 expression and SA levels in cbp60g-1 snc2-1D are suppressed by bda7-1 (Figure 1C-1E), we examined whether the expression of SARD1 is affected in the $c b p 60 b$ cbp60g-1 snc2-1D mutants. As shown in Figure 3A, the expression level of SARD1 is much higher in $\operatorname{cbp} 60 g-1$ snc2-1D than in wild type, and the elevated SARD1 expression is fully suppressed in $c b p 60 b$ cbp60g-1 snc2-1D, suggesting that CBP60b functions upstream of SARD1. We also examined the expression levels of FMO1, which is another target gene of SARD1. Consistent with the expression levels of SARD1, up-regulation of FMO1 in cbp60g-1 snc2-1D is also blocked in cbp60b cbp60g-1 snc2-1D (Figure 3B). These data suggest that CBP60b functions upstream of SARD1. 
As CBP60b is predicted to be a transcription factor, we tested whether CBP60b binds to the promoter of SARD1. ChIP-qPCR assay was carried out on transgenic plants expressing CBP60b$3 H A$ under its own promoter in the cbp60b-1 cbp60g-1 snc2-1D background. Compared to the non-transgenic negative control cbp60g-1 snc2-1D, 4.6-fold enrichment of the DNA around 1.0 $\mathrm{kb}$ upstream of the start codon and $\sim 2.8$ fold enrichment of the DNA around $0.3 \mathrm{~kb}$ upstream of the start codon were observed in the samples from the CBP6Ob-3HA transgenic line (Figure 3C), indicating that $\mathrm{CBP} 60 \mathrm{~b}$ is recruited to the $S A R D 1$ promoter region.

To further test whether CBP60b can activate the expression of SARD1, a plasmid expressing a luciferase reporter gene under the control of the SARD1 promoter ( $p S A R D 1:: L u c$ ) was transformed into Nicotiana (N.) benthamiana, together with a 35S::CBP60b-3HA construct. Cotransformation of $35 S:: C B P 60 b-3 H A$ with $p S A R D 1:: L u c$ resulted in increased expression of the luciferase reporter (Figure 3D and 3E), suggesting that overexpression of $C B P 60 b$ in $N$. benthimiana leads to activation of SARD1 expression. Together, these data suggest that CBP60b serves as a transcriptional activator of $S A R D 1$.

\section{Overexpression of $C B P 60 \mathrm{~b}$ leads to up-regulation of $S A R D 1$ and enhanced disease resistance in Arabidopsis}

To further test the hypothesis that CBP60b regulates $S A R D 1$ expression, we overexpressed CBP60b in wild type Col-0 background. As shown in Figure 4A, transgenic plants overexpressing CBP6Ob-3HA exhibit dwarfism with dark green leaves. The expression levels of $S A R D 1$ are significantly higher in the $C B P 60 b-3 H A$ transgenic lines than in the wild type control (Figure 4B), further supporting the role of CBP60b in regulating SARD1 expression. Consistent with the elevated SARD1 expression levels, these transgenic lines showed enhanced resistance to Hpa Noco2 (Figure 4C).

\section{cbp60b single mutants exhibit constitutively activated immune responses}

The requirement of CBP60b for the constitutive defense responses in $c b p 60 g-1$ snc2-1D indicates that $\mathrm{CBP} 60 \mathrm{~b}$ serves as a positive regulator of plant immunity. To our surprise, when we isolated the $c b p 60 b-1$ single mutant from the F2 population of a cross between $c b p 60 b-1$ cbp60g- 
1 snc2-1D and Col-0, the cbp60b-1 plants displayed a dwarf morphology with dark green and abnormal leaves (Figure S8). cbp60b-1 cbp60g-1 double mutant plants isolated from the same F2 population showed an even more dramatic phenotype, as they were seedling-lethal and grew only two cotyledons at room temperature (Figure S8). To rule out the possibility that the unexpected phenotypes originated from random background mutations in cbp60b-1 cbp60g-1 snc2-1D, we generated $c b p 60 b$ deletion mutants in wild type Col-0 and $\operatorname{cbp} 60 g-1$ backgrounds by CRISPR/Cas9. As shown in Figure 5A, the cbp60b deletion mutants showed almost identical morphology as the corresponding cbp60b-1 mutants in wild type and cbp60g- 1 backgrounds, confirming that the dwarfism observed in cbp60b-1 and cbp60b-1 cbp60g-1 is caused by the mutation in $C B P 60 b$.

Next, we tested whether defense responses are activated in these mutants. As shown in Figure $5 \mathrm{~B}$, the expression levels of $P R 1$ were dramatically increased in $c b p 60 b$ mutants, and even higher in the $c b p 60 b$ cbp60g-1 double mutants. cbp60b and cbp60b cbp60g-1 mutants also exhibited elevated ICS1 expression (Figure 5C). Consistently, SA levels in cbp60b mutants were much higher than in the wild type (Figure 5D and 5E). In addition, $c b p 60 b$ mutants showed strong resistance against $\mathrm{Hpa}$ Noco2 (Figure 5F). These observations suggest that knocking out $C B P 60 b$ leads to constitutive activation of defense responses.

We further examined whether mutations in $S A R D 1$ affects the autoimmunity of cbp60b cbp60g1. cbp60b cbp60g-1 sard1-1 triple mutants were generated through knocking out CBP60b in the cbp60g-1 sard1-1 double mutant by CRISPR/Cas9. Similar to the cbp60b cbp60g-1 double mutant, cbp60b cbp60g-1 sard1-1 triple mutants were seedling lethal and grew only two cotyledons (Figure S9A). Unlike cbp60b cbp60g-1, cotyledons of cbp60b cbp60g-1 sard1-1 plants also showed visible lesion (Figure S9A).

Positive regulators of plant immunity are often monitored/guarded by NLR receptors (Cui et al., 2015). To test whether the constitutive defense responses in $c b p 60 b$ is caused by activation of NLR-mediated immunity, we crossed $c b p 60 b-2$ with eds1-24, a CRISPR deletion knockout mutant of EDS1, a gene known to be required for defense signaling mediated by Toll/interleukin1 receptor (TIR) domain containing NLRs (TNLs)(Aarts et al., 1998). The cbp60b-2 eds1-24 
double mutant showed wild type-like morphology (Figure 5G). In addition, the constitutive PRI expression and enhanced resistance to $\mathrm{Hpa}$ Noco2 are completely suppressed in $c b p 60 b-2$ eds 1 24 (Figure 5H and 5I), indicating that the autoimmunity of $c b p 60 b$ is dependent on EDS1, and TNL-mediated immunity is likely activated with loss of CBP6Ob function.

As NLR-mediated autoimmunity can often be suppressed by high temperature (van Wersch et $a l ., 2016)$, we tested whether the dwarf phenotype of $c b p 60 b, c b p 60 b c b p 60 g-1$ and $c b p 60 b$ cbp60g-1 sard1-1 can be suppressed by growing them at $28^{\circ} \mathrm{C}$. As shown in Figure $\mathrm{S} 9 \mathrm{~B}, \operatorname{cbp} 60 b$ exhibited wild type morphology at $28^{\circ} \mathrm{C}$, while cbp60b cbp60g-1 and cbp60b cbp60g-1 sard1-1 plants had intermediate size and were able to grow true leaves at $28{ }^{\circ} \mathrm{C}$.

\section{Discussion}

SARD1 and CBP60g play critical roles in transcriptional regulation of plant immunity (Sun et al., 2015). Unlike CBP60g, which requires binding of CaM for its activation (Wang et al., 2009), SARD1 is primarily regulated at transcription level. The autoimmune mutant $s n c 2-1 D$ provides a unique system to uncover components regulating $S A R D$ lexpression, as the constitutive defense responses in cbp60g-1 snc2-1D is mainly dependent on SARD1 (Sun et al., 2015). From a suppressor screen of $c b p 60 g-1 s n c 2-1 D$, here we identified CBP60b as a positive regulator of $S A R D 1$ transcription. CBP60b is targeted to the promoter region of $S A R D 1$ and required for its up-regulation in $c b p 60 g-1$ snc2-1D. Overexpression of $C B P 60 b$ leads to elevated SARD1 expression and constitutive defense responses. In addition, transient expression of $C B P 60 \mathrm{~b}$ in $N$. benthamiana activates the expression of the pSARD1-Luc reporter gene, confirming that CBP60b positively regulates the expression of $S A R D 1$.

CBP60b belongs to the same protein family as SARD1 and CBP60g (Reddy et al., 2002), which share a highly conserved central domain with DNA-binding activity but have divergent sequences at the $\mathrm{N}$ - and C-termini (Wang et al., 2009, Zhang et al., 2010a). Unlike SARD1 and CBP60g, which are strongly induced during pathogen infection (Wang et al., 2009, Zhang et al., 2010a), CBP60b is constitutive expressed in different tissue (Reddy et al., 2002). CBP60b was identified as a CaM-binding protein, suggesting that its activity might be influenced by $\mathrm{Ca}^{2+}$ levels (Reddy et al., 2002). SARD1 was previously shown to activate ICS1 expression through a 
GAAATTT motif on its promoter (Sun et al. 2015). CBP60b shares $\sim 80 \%$ sequence similarity with SARD1 in the middle domain. Whether it binds to a similar DNA sequence in activating SARD1 expression remains to be determined.

Surprisingly, defense responses are constitutively activated in the $c b p 60 b$ single mutants. The constitutive defense response in $c b p 60 b$ is blocked by knocking out $E D S 1$, suggesting that loss of $C B P 60 \mathrm{~b}$ leads to activation of TNL-mediated immune signaling. It is possible that CBP60b is required for the expression of a negative regulator of TNL-mediated immune signaling. More likely, CBP60b may be monitored by a TNL and loss of its function triggers activation of this unidentified TNL and downstream defense responses, since critical immune regulators are often targeted by pathogen effector proteins and guarded by plant NLR receptors (Cui et al., 2015, Kourelis and Van Der Hoorn, 2018). Although no pathogen effector has been reported to target CBP60b, SARD1 and CBP60g were shown to be targets of Verticillium effector VdSCP4 (Qin et al., 2018).

Compared to $c b p 60 b$, the autoimmune phenotype of the $c b p 60 b$ cbp60g- 1 double mutants is even more dramatic, suggesting that $C B P 60 b$ and $C B P 60 g$ play partially redundant roles in transcriptional regulation of the putative guardee/decoy recognized by the unknown TNL. In $c b p 60 b$, the expression of the putative guardee/decoy is partially blocked, leading to activation of TNL-mediated immunity. Lacking both CBP60b and CBP60g leads to further reduction of its expression and stronger defense responses. Interestingly, the severe dwarf phenotype of $c b p 60 b$ cbp60g- 1 was not observed in the $s n c 2-1 D$ background. It is possible that $s n c 2-1 D$ can also activate CBP60b and CBP60g-inpendent expression of the putative guardee/decoy, which compensates the loss of CBP60b and CBP60g.

A working model is proposed based on our findings (Figure 6). CBP60b promotes the expression of SARD1 and contributes to SNC2-mediated immunity. It is required for the expression of a yetto-be-identified guardee/decoy of a TNL. Disruption of the expression of the putative guardee/decoy results in activation of TNL-mediated immunity. It is of future interest to identify this guardee/decoy and the corresponding TNL. 


\section{Materials and Methods}

\section{Plant materials and growth conditions}

All Arabidopsis thaliana mutants are in the Col-0 ecotype background unless specified. The cbp60g-1 snc2-1D, cbp60g-1, cbp60g-1 sard1-1 plants were described previously (Zhang et al., 2010a, Sun et al., 2015). cbp60b-1 and cbp60b-1 cbp60g-1 plants were isolated from a cross between cbp60b-1 cbp60g-1 snc2-1D and Col-0. cbp60b-2, cbp60b-3 are two independent deletion lines generated by CRISPR/Cas9. The CBP6Ob-3HA-OX \#21 and \#27 are two independent overexpression lines generated by transforming Col-0 with Agrobacteria carrying pCambia1300-35S-CBP60b-3HA. eds1-24 is a CRISPR mutant line with deleting both EDS1A and $E D S 1 B$ genes, as described previously (Tian et al., 2020). cbp60b-2 eds1-24 was obtained from the F2 population of a cross between $c b p 60 b-2$ and $e d s 1-24$. Primers used for genotyping were listed in Table S1.

Plants were grown on soil under long-day conditions (16-h light/ 8-h dark cycle) with a light intensity of $\sim 100 \mu \mathrm{mol} / \mathrm{m}^{2} / \mathrm{s}$ of at $22{ }^{\circ} \mathrm{C}$ unless specified. Plants for quantitative RT-PCR were grown on plates with $1 / 2$ Murashige and Skoog (MS) and 1\% sucrose for two weeks. Plants used for SA quantification were grown on soil for four weeks under short-day conditions (8-h light/ 16-h dark cycle).

\section{Constructs for generation of deletion mutants and transgenic plants}

The CRISPR/Cas9 system used for generating cbp60b mutants was described previously (Xing et al., 2014). Two guide RNAs were designed to target CBP6Ob genomic DNA for generation of a deletion of $\sim 1 \mathrm{~kb}$ in size. A PCR fragment containing the guide RNA sequences was amplified from the pCBC-DT1T2 vector using primers At5g57580-BsFF0 and At5g57580-BsRR0 and subsequently inserted into the pHEE401 vector using the BsaI site. The derived plasmid was transformed into E. coli DH10B and later Agrobacterium GV3101 by electroporation.

Arabidopsis plants were transformed with the Agrobacterium carrying the plasmid by floral dipping (Clough and Bent, 1998). T1 plants were analyzed for deletion in CBP60b by PCR with primers listed in Table S1. Homozygous deletion mutants were obtained in the T2 generation. 
To overexpress $C B P 60 b, C B P 60 b$ was amplified from genomic DNA using primers AT5G57580-atgKpnI-F and AT5G57580-nstopBamHI-R. The PCR fragment was digested with KpnI and BamHI and afterwards ligated into pCambia1300-35S-3HA vector. The derived plasmid was transformed into E. coli DH10B and later Agrobacterium GV3101. Wild type Col-0 plants were transformed with Agrobacterium carrying the plasmid by floral dipping. Homozygous transgenic lines were obtained in the T2 generation.

For transgene complementation, a genomic DNA fragment containing $C B P 60 b$ was amplified with primers AT5G57580-KpnI-F and AT5G57580-nstopBamHI-R. The PCR fragment was inserted into a pBasta-3HA vector derived from pCambia1305 to obtain the pAt5g57580::At5g57580-3HA construct. The pAt5g57580::At5g57580-3HA/cbp60b-1 cbp60g-1 snc2-1D transgenic lines were obtained by transforming cbp60b-1 cbp60g-1 snc2-1D plants with Agrobacterium carrying the plasmid of pAt5g57580::At5g57580-3HA.

\section{Chromatin immunoprecipitation (ChIP) analysis}

ChIP-qPCR assays were performed as previously described (Sun et al., 2015). The chromatin complexes containing CBP60b-3HA were immunoprecipitated using anti-HA antibody (Roche, Basel, Switzerland) and Protein A/G Agarose beads (GE Healthcare, Chicago, United States). The immunoprecipitated DNA was analyzed by qPCR using gene specific primers, which were listed in Table S1.

\section{Genetic mapping and identification of $b d a 7-1$}

Genetic mapping of $b d a 7-1$ was carried out on the F2 population of a cross between $b d a 7-1$ cbp60g-1 snc2-1D mutant (in Col-0 background) and Landsberg erecta (Ler). Afterwards, Illumina sequencing was used to identify the bda7-1mutation. bda7-1 cbp60g-1 snc2-1D was backcrossed with cbp60g-1 snc2-1D. Genomic DNA from 50 F2 plants with similar morphology as bda7-1 cbp60g-1 snc2-1D were pooled and sequenced.

\section{Dual reporter assay}

Dual reporter assay was performed in Nicotiana benthamiana by transforming the reporter constructs together with the different effector constructs. The $p S A R D 1:: L u c$ were described 
previously (Ding et al., 2018). A pUBQ1-driven Renilla luciferase reporter was included as internal control. Agrobacteria carrying the reporter construct $p S A R D 1:: L u c$, internal control pUBQ1-Renilla and effector construct $35 S:: C B P 60 b-3 H A$ or $35 S:: G F P-3 H A$ were first cultured in liquid $\mathrm{LB}$ and then resuspended in $10 \mathrm{mM} \mathrm{MgCl}_{2}$. Leaves of $N$. benthamiana were coinfiltrated with Agrobacteria carrying the indicated construct combinations with final concentrations of $\mathrm{OD}_{600}=0.2(p S A R D 1:: L u c), \mathrm{OD}_{600}=0.05(p U B Q 1-$ Renilla $)$ and $\mathrm{OD}_{600}=0.5$ (35S::CBP60b-3HA or 35S::GFP-3HA). For imaging the luminescence intensity, $N$. benthamiana leaves was infiltrated with $1 \mathrm{mM}$ luciferin $40 \mathrm{~h}$ after inoculation of the Agrobacteria and then imaged under the Gel Doc XR+ System (Bio-Rad) with the Bolt mode. For quantification of the promoter activity, areas of $N$. benthamiana leaves inoculated with Agrobacteria was collected $40 \mathrm{~h}$ after inoculation. The Dual-Luciferase ${ }^{\circledR}$ Reporter Assay System (Promega) was used to measure the activity of firefly luciferase and renilla luciferase sequentially using a BioTek ${ }^{\mathrm{TM}}$ Synergy $^{\mathrm{TM}} 2$ Multi-Mode Microplate Reader. Relative promoter activities were calculated as the ratio of firefly luciferase/renilla luciferase.

\section{RNA extraction, reverse transcription and qPCR}

Plants for gene expression assays were grown on $1 / 2$ MS plates for 14 days under long-day conditions. Approximately $50 \mathrm{mg}$ plant tissue from 3-4 individual seedlings of the indicated genotypes were collected as a single sample. Three biological replicates were analyzed for each genotype. RNA extraction was performed using the EZ-10 Spin Column Plant RNA Miniprep Kit (Bio Basic Inc., Toronto, Canada). RNAs were reverse transcribed into cDNAs by OneScript Reverse Transcriptase (Applied Biological Materials Inc., Richmond, Canada). qPCR was performed on the total cDNAs using SYBR Premix Ex TaqTM II (Takara, Shiga, Japan). Primers for qPCR are listed in Table S1.

\section{SA extraction and quantification}

The procedure of SA extraction and measurement was reported previously (Sun et al., 2015). 100 mg of plant tissue was collected from 2-3 individual plants of the indicated genotypes as a single sample. Plant tissue was ground into fine powder with liquid nitrogen, resuspended with $600 \mu 190 \%$ methanol and sonicated for $20 \mathrm{~min}$ to release SA. After centrifugation at 12,000×g for $10 \mathrm{~min}$, the supernatant was collected, and the pellets underwent a second round of extraction 
by adding $500 \mu \mathrm{l}$ of $100 \%$ methanol and sonicating for another $20 \mathrm{~min}$. The supernatant from both extractions were combined together and dried by vacuum. Next, $500 \mu \mathrm{l}$ of $5 \%(\mathrm{w} / \mathrm{v})$ trichloroacetic acid was added to the dry samples, vortexed and sonicated for $5 \mathrm{~min}$. After centrifugation at $12,000 \mathrm{~g}$ for $15 \mathrm{~min}$, the supernatant was collected and extracted with $500 \mu \mathrm{l}$ extraction buffer (ethylacetate acid: cyclopentane: isoporopanal, 100:99:1, v/v/v) for three times. Each time, after centrifugation at $12,000 \times \mathrm{g}$ for $10 \mathrm{~min}$, the organic phase was collected and combined into a new tube. The combined organic phase was then dried by vacuum. The dry sample was next resuspended with $200 \mu \mathrm{l}$ mobile phase (0.2M KAc, 0.5mM EDTA pH=5) by vortexing and sonicating for $5 \mathrm{~min}$. After the final centrifugation at $12,000 \times \mathrm{g}$ for $5 \mathrm{~min}$, the supernatant was collected and used to determine the amount of SA by high-performance liquid chromatography.

\section{Pathogen infection assay}

Hpa Noco2 infection was conducted by spray-inoculating two-week-old seedlings with spores in water $(50,000$ spores $/ \mathrm{mL})$. Inoculated seedlings were covered with a transparent lid and grown in a plant chamber at $18{ }^{\circ} \mathrm{C}$ with a relative humidity of $\sim 80 \%$. Infection was scored at $7 \mathrm{dpi}$ by counting conidia spores with a hemocytometer. 4-5 individual plants were pooled as a single sample. 4 biological replicates were included for each genotype.

\section{Statistical analysis}

Error bars in all of the figures represent standard deviations. The number of biological replicates is indicated in the figure legends. Statistical comparison among different samples is carried out by either one-way ANOVA with Tukey's honestly significant difference (HSD) post hoc test or Student's t-test, as reported in the figure legends.

\section{Acknowledgement}

We would like to acknowledge Dr. Tongjun Sun for suggestions on the ChIP assay. We are grateful to Sean Shang for his help with the NGS data pipeline. We thank Jia Yao for her technical supports on the project. We thank Dr. Yanan Liu, Dr. Xingchuan Huang and Dr. Wei Li for the help with handling the sequencing samples. 


\section{Funding}

We are grateful for the financial support to Y. Z. and X. L. from the Natural Sciences and Engineering Research Council (NSERC) Discovery Program of Canada. W. H. was supported by the China Scholarship Council and NSERC-CREATE (PRoTECT).

\section{Author contributions}

YZ and XL designed and supervised the study. WH, WZ and HT conducted the experimental research. $\mathrm{WH}, \mathrm{XL}$ and $\mathrm{YZ}$ analyzed the data and wrote the manuscript. 
A

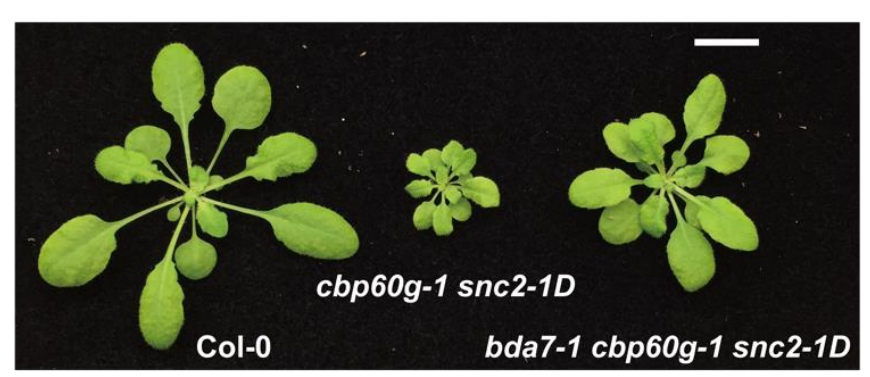

B

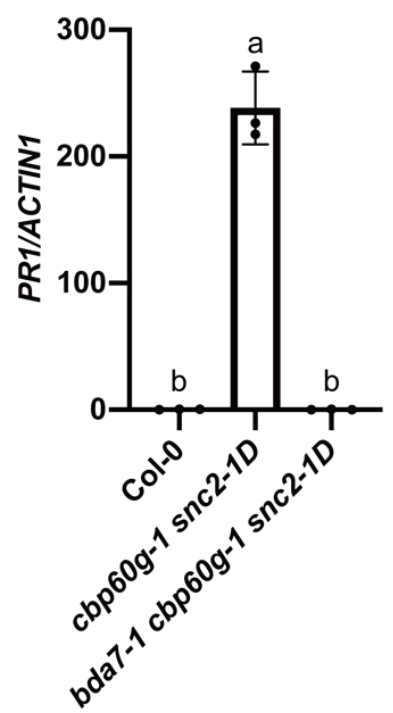

C

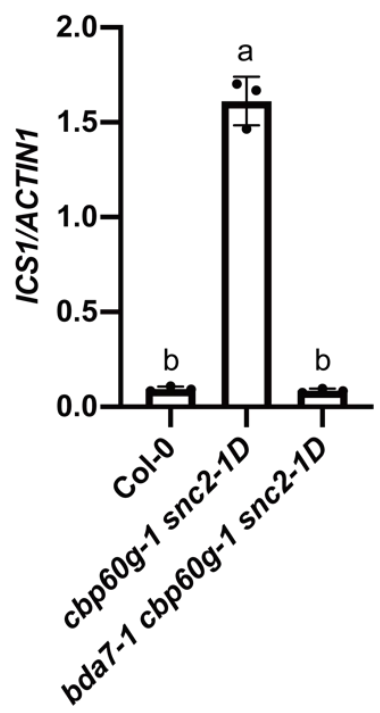

D

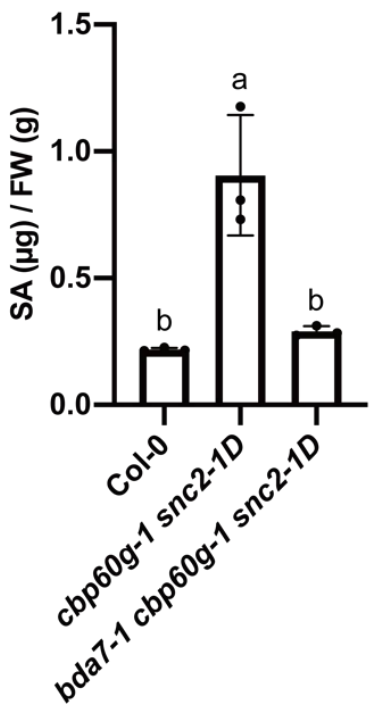

E

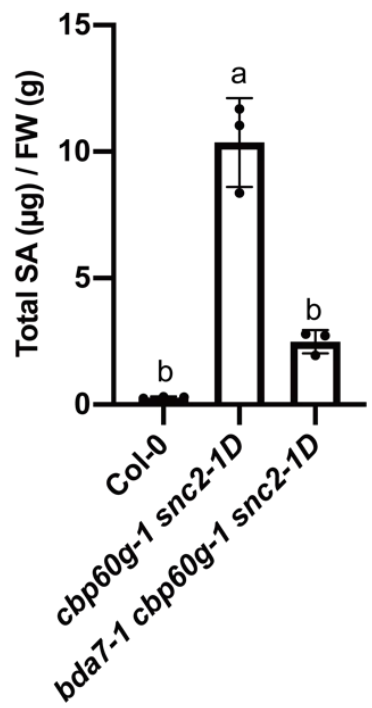

$\mathbf{F}$

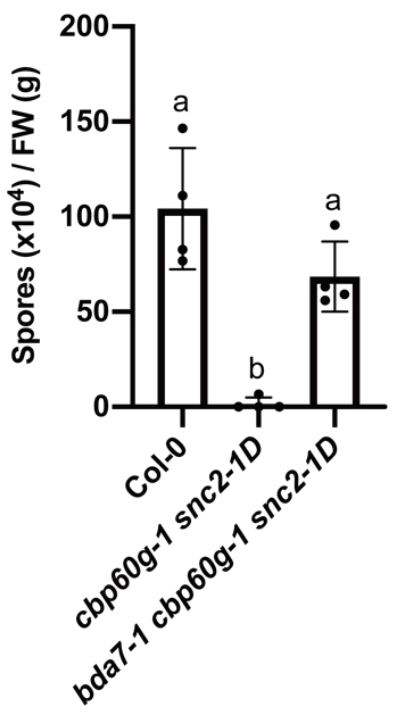

Figure 1. Identification and characterization of bda7-1 cbp60g-1 snc2-1D.

(A) Morphology of four-week-old soil-grown plants of Col-0, cbp60g-1 snc2-1D and bda7-1 cbp60g-1 snc2-1D under long-day condition. Scale bar is $1 \mathrm{~cm}$.

(B and C) Relative expression levels of PRI (B) and ICS1 (C) in the indicated genotypes.

Transcript levels were normalized with ACTIN1. Error bars represent standard deviations. Letters indicate statistical differences $(\mathrm{P}<0.0001$, one-way ANOVA followed by Tukey's multiple comparisons test; $\mathrm{n}=3$ ).

(D, E) Free (D) and total SA (E) levels in the indicated genotypes. Error bars represent standard deviations. Letters indicate statistical differences $(\mathrm{P}<0.01$, one-way ANOVA followed by Tukey's multiple comparisons test; $\mathrm{n}=3$ ). 
bioRxiv preprint doi: https://doi.org/10.1101/2021.04.15.440066; this version posted April 16, 2021. The copyright holder for this preprint (which

was not certified by peer review) is the author/funder, who has granted bioRxiv a license to display the preprint in perpetuity. It is made available under aCC-BY-NC-ND 4.0 International license.

(F) Growth of $\mathrm{Hpa}$ Noco2 conidiospores on the indicated genotypes. Error bars represent standard deviations. Letters indicate statistical differences $(\mathrm{P}<0.01$, one-way ANOVA followed by Tukey's multiple comparisons test; $n=4$ ). 
A

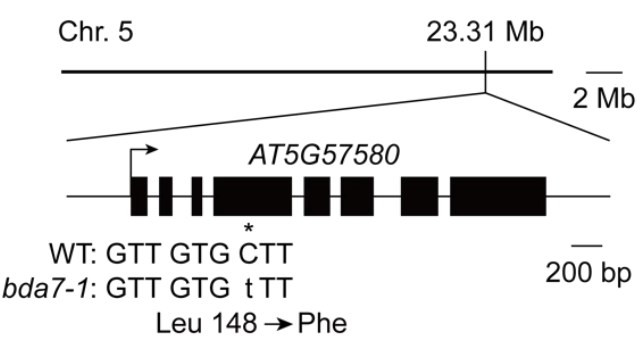

B

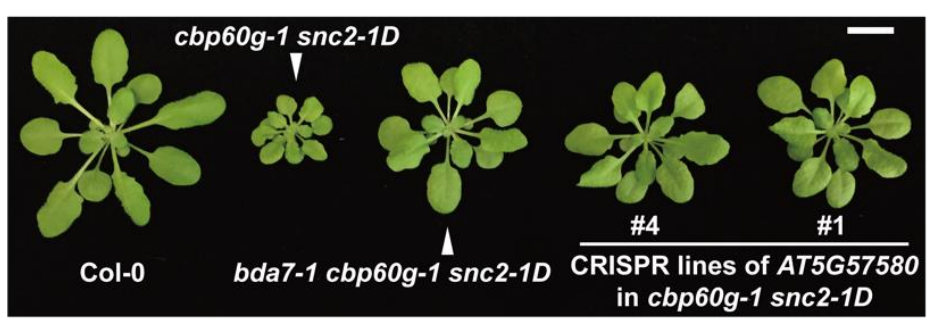

E

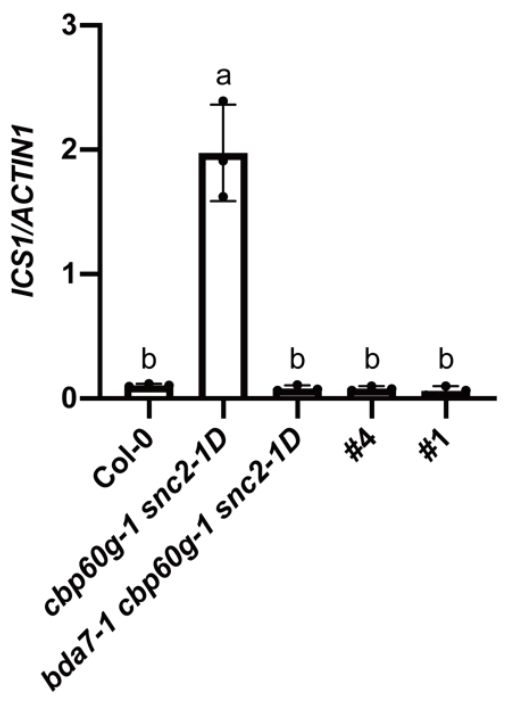

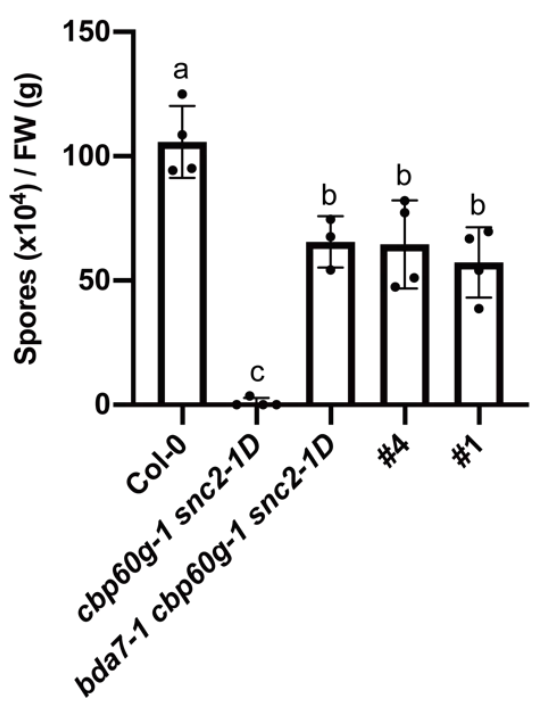

Figure 2. Deletion mutants of AT5G57580 suppress the constitutively activated immunity of cbp60g-1 snc2-1D.

(A) Map position and the mutation in $b d a 7-1$. The asterisk is used to mark the position of $b d a 7$ 1 .

(B) Morphology of four-week-old soil-grown plants of the indicated genotypes under long-day condition. Scale bar is $1 \mathrm{~cm}$.

(C and D) Expression levels of PRI (C) and ICS1 (D) in the indicated genotypes as normalized by ACTIN1. Error bars represent standard deviations. Letters indicate statistical differences $(\mathrm{P}<$ 0.01 , one-way ANOVA followed by Tukey's multiple comparisons test; $\mathrm{n}=3$ ).

(E) Growth of $\mathrm{Hpa} \mathrm{Noco} 2$ conidiospores on the indicated genotypes. Error bars represent standard deviations. Letters indicate statistical differences $(\mathrm{P}<0.01$, one-way ANOVA followed by Tukey's multiple comparisons test; $\mathrm{n}=4$ ). 
A

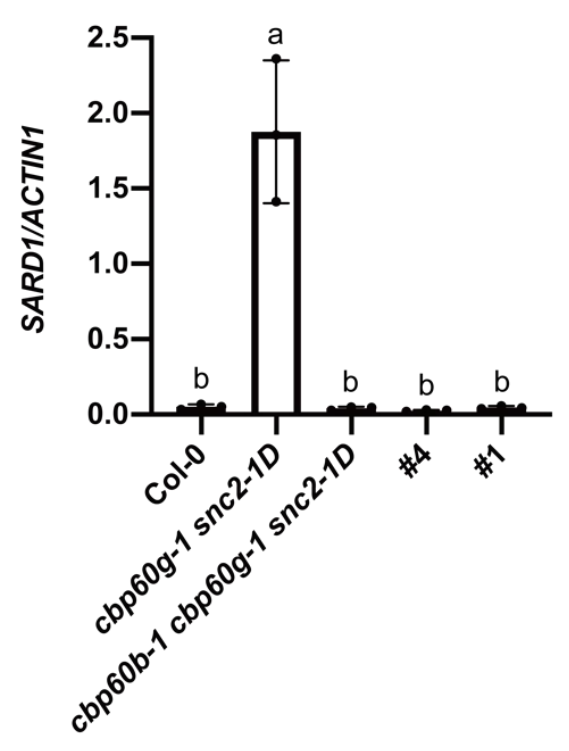

B

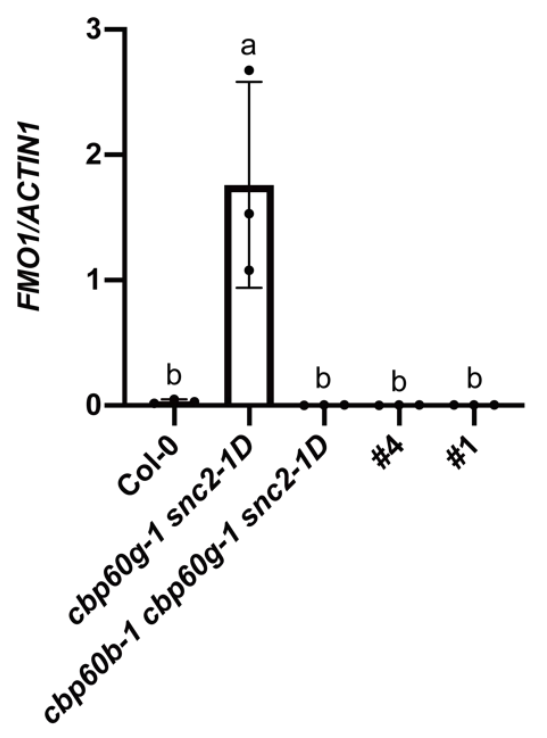

C

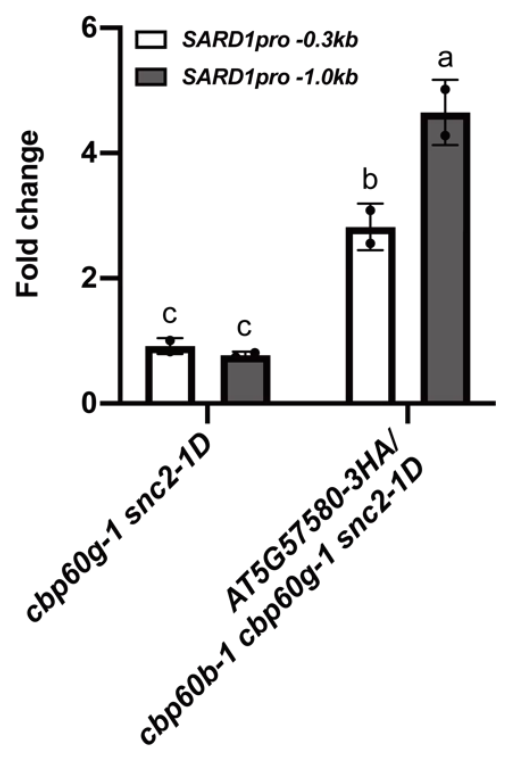

E

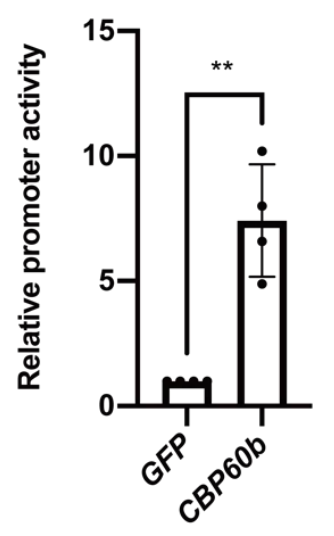

Figure 3. Regulation of $S A R D 1$ expression by CBP60b.

(A and B) Expression levels of SARD1 (A) and FMO1 (B) in the indicated genotypes as normalized by ACTIN1. Error bars represent standard deviations. Letters indicate statistical differences ( $\mathrm{P}<0.01$, one-way ANOVA followed by Tukey's multiple comparisons test; $\mathrm{n}=3$ ). (C) Binding of CBP60b to the promoter of SARD1 as determined by ChIP-PCR. Two-week-old seedlings grown on $1 / 2 \mathrm{MS}$ plates were sprayed with $1 \mu \mathrm{M}$ of the elicitor nlp20 $5 \mathrm{~h}$ before tissue collection. CBP60b-3HA chromatin complexes were immunoprecipitated with anti-HA antibody and protein $\mathrm{G}$ agarose beads. Negative control reactions were performed in parallel without adding anti-HA antibody. Immunoprecipitated DNA samples were quantified by qPCR using primers specific to $S A R D 1$ promoter. ChIP results are presented as fold changes by dividing signals from ChIP with the anti-HA antibody by those from no antibody controls. Error bars represent standard deviations. Letters indicate statistical differences $(\mathrm{P}<0.05$, one-way ANOVA followed by Tukey's multiple comparisons test; $\mathrm{n}=2$ ).

(D) Activation of pSARD1::Luc reporter gene expression by CBP60b in $N$. benthamiana leaves. Agrobacteria carrying $p S A R D 1:: L u c\left(\mathrm{OD}_{600}=0.2\right)$ and $35 S: \because R l u c$ expressing the Renilla luciferase $\left(\mathrm{OD}_{600}=0.05\right)$ were co-infiltrated with Agrobacteria carrying $35 S:: C B P 60 b$ or 
$35 S:: G F P\left(\mathrm{OD}_{600}=0.5\right)$. The left diagram illustrates the different treatments. 2 days after inoculation, luminescence was detected after infiltration with $1 \mathrm{mM}$ luciferin, as shown in the right image.

(E) Quantification of firefly luciferase activities in N. benthamiana leaves co-transformed with the construct combinations as indicated in (D). Relative promoter activity was presented as a ratio of firefly luciferase/renilla luciferase. Relative promoter activity of the GFP control group was set as 1. ${ }^{* *}$ indicates statistical differences $(\mathrm{P}<0.01$, unpaired $\mathrm{t}$ test; $\mathrm{n}=4)$. 
A

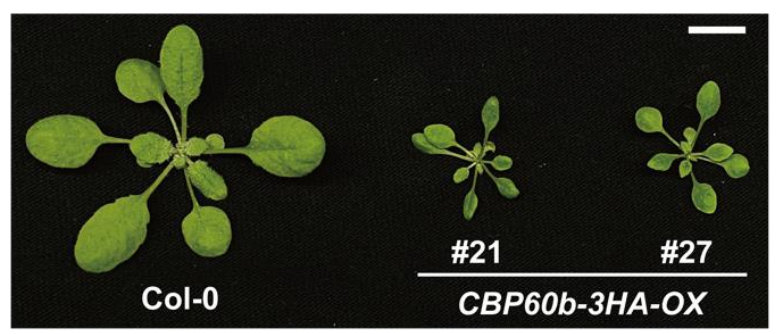

B

C
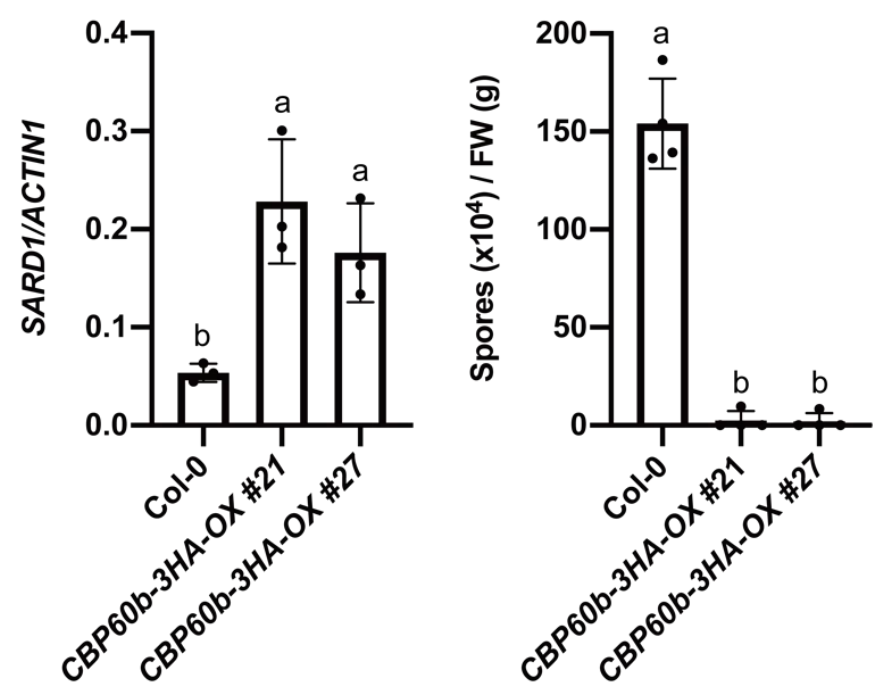

Figure 4. Overexpression of $C B P 60 b$ leads to increased $S A R D 1$ expression and enhanced resistance against $\mathrm{Hpa}$ Noco2.

(A) Morphologies of three-week-old soil-grown plants of the indicated genotypes under long-day condition. Scale bar is $1 \mathrm{~cm}$.

(B) Expression level of SARDI in the indicated genotypes as normalized by ACTIN1. Error bars represent standard deviations. Letters indicate statistical differences $(\mathrm{P}<0.05$, one-way ANOVA followed by Tukey's multiple comparisons test; $\mathrm{n}=3$ ).

(C) Growth of $\mathrm{Hpa} \mathrm{NocO} 2$ on the indicated genotypes. Error bars represent standard deviations. Letters indicate statistical differences $(\mathrm{P}<0.0001$, one-way ANOVA followed by Tukey's multiple comparisons test; $\mathrm{n}=4$ ). 


\section{A}

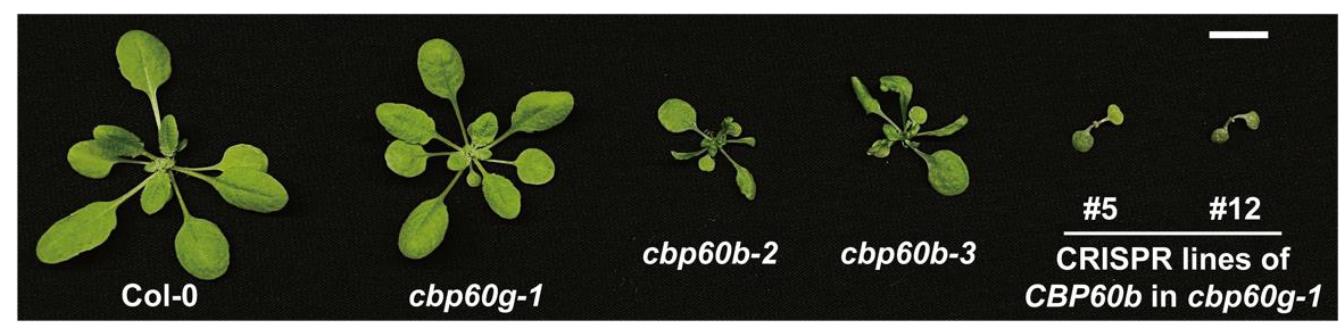

B
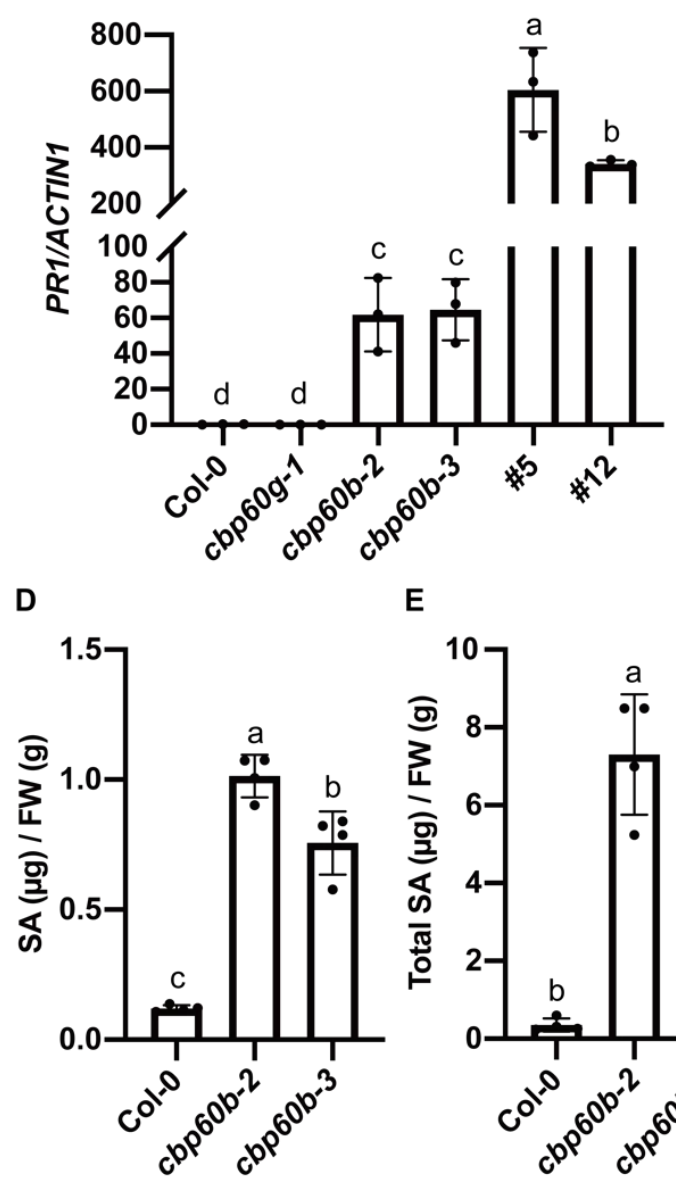

E

C

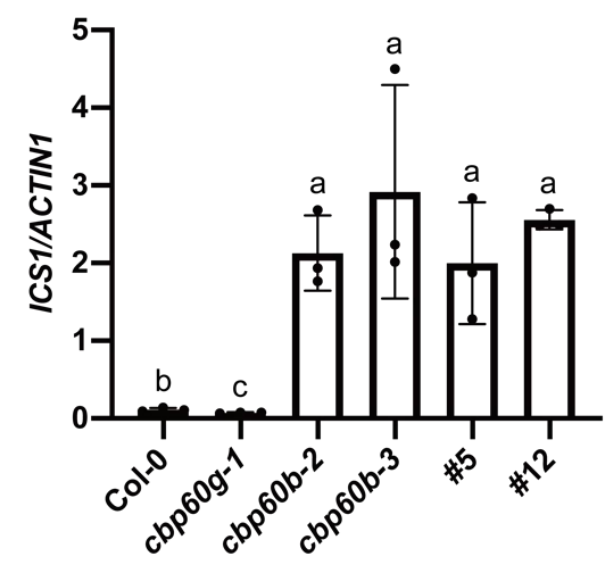

F

G

H
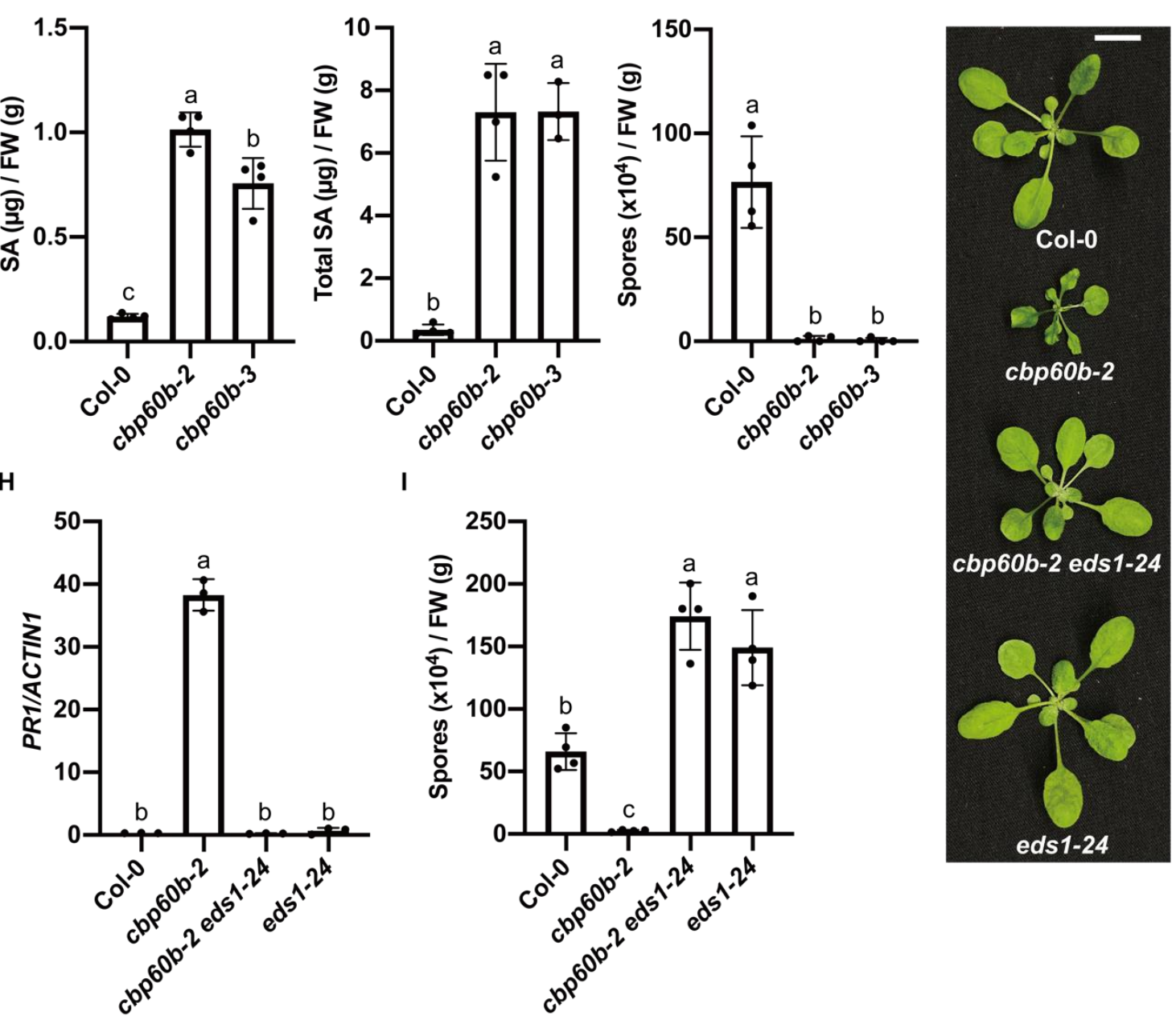
Figure 5. Knocking out $C B P 60 b$ activates $E D S 1$-dependent defense responses.

$(A, G)$ Morphologies of three-week-old soil-grown plants of the indicated genotypes under longday condition. Scale bar is $1 \mathrm{~cm}$.

$(\mathrm{B}, \mathrm{C})$ Expression levels of $P R 1(\mathrm{~B})$ and $\operatorname{ICS1}(\mathrm{C})$ in the indicated genotypes as normalized by those of ACTIN1. Error bars represent standard deviations. Letters indicate statistical differences $(\mathrm{P}<0.05$, Student's t-test; $\mathrm{n}=3$ ).

$(\mathrm{D}, \mathrm{E})$ Free (D) and total SA(E) levels in the indicated genotypes. Error bars represent standard deviations. Letters indicate statistical differences $(\mathrm{P}<0.01$, one-way ANOVA followed by Tukey's multiple comparisons test; $n=4$ ).

(F) Growth of Hpa Noco2 on the indicated genotypes. Error bars represent standard deviations. Letters indicate statistical differences ( $\mathrm{P}<0.0001$, one-way ANOVA followed by Tukey's multiple comparisons test; $\mathrm{n}=4$ ).

(H) Expression levels of PRI in the indicated genotypes as normalized by those of ACTIN1. Error bars represent standard deviations. Letters indicate statistical differences $(\mathrm{P}<0.0001$, oneway ANOVA followed by Tukey's multiple comparisons test; $\mathrm{n}=3$ ).

(I) Growth of $\mathrm{Hpa}$ Noco 2 on the indicated genotypes. Error bars represent standard deviations. Letters indicate statistical differences $(\mathrm{P}<0.01$, one-way ANOVA followed by Tukey's multiple comparisons test; $\mathrm{n}=4$ ). 


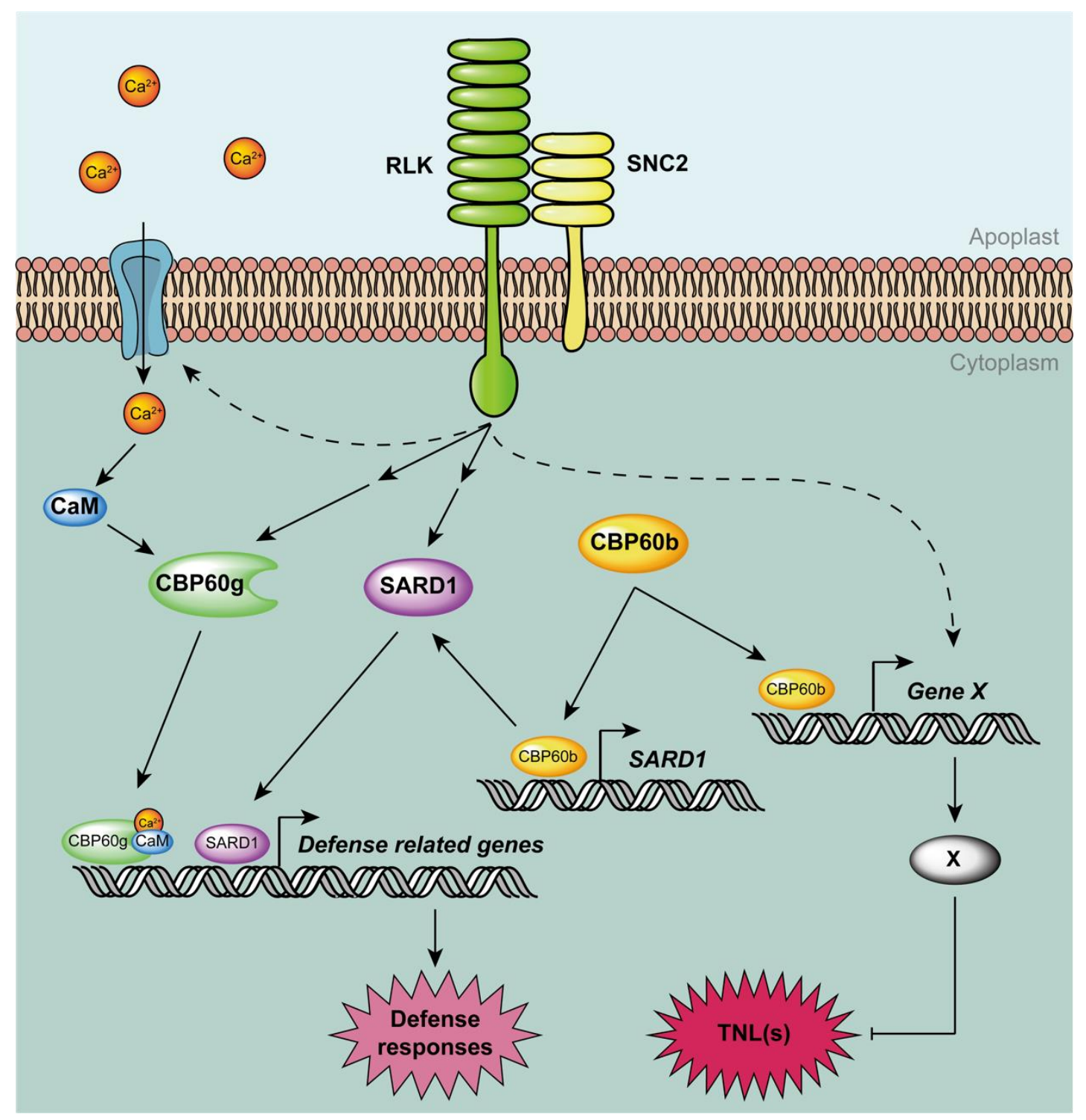

Figure 6. A working model of CBP60b in plant immunity. On one hand, CBP60b contributes to SNC2-mediated immunity by promoting SARD1 expression. On the other hand, it is also required for the expression of an unknown gene (X), which encodes a guardee/decoy of a TNL(s) or a negative regulator of TIR signaling. Activation of SNC2-mediated defense responses also leads to CBP60b-independent expression of gene X. 


\section{Reference:}

Aarts, N., Metz, M., Holub, E., Staskawicz, B.J., Daniels, M.J. and Parker, J.E. (1998) Different requirements for EDS1 and NDR1 by disease resistance genes define at least two $R$ gene-mediated signaling pathways in Arabidopsis. Proc Natl Acad Sci U S A, 95, 1030610311.

Clough, S.J. and Bent, A.F. (1998) Floral dip: a simplified method for Agrobacterium-mediated transformation of Arabidopsis thaliana. Plant J, 16, 735-743.

Cui, H., Tsuda, K. and Parker, J.E. (2015) Effector-triggered immunity: from pathogen perception to robust defense. Annu Rev Plant Biol, 66, 487-511.

Ding, Y., Sun, T., Ao, K., Peng, Y., Zhang, Y., Li, X. and Zhang, Y. (2018) Opposite Roles of Salicylic Acid Receptors NPR1 and NPR3/NPR4 in Transcriptional Regulation of Plant Immunity. Cell, 173, 1454-1467 e1415.

Dou, D. and Zhou, J.-M. (2012) Phytopathogen effectors subverting host immunity: different foes, similar battleground. Cell host \& microbe, 12, 484-495.

Kourelis, J. and Van Der Hoorn, R.A. (2018) Defended to the nines: 25 years of resistance gene cloning identifies nine mechanisms for R protein function. The Plant cell, 30, 285-299.

Monaghan, J. and Zipfel, C. (2012) Plant pattern recognition receptor complexes at the plasma membrane. Curr Opin Plant Biol, 15, 349-357.

Qin, J., Wang, K., Sun, L., Xing, H., Wang, S., Li, L., Chen, S., Guo, H.-S. and Zhang, J. (2018) The plant-specific transcription factors CBP60g and SARD1 are targeted by a Verticillium secretory protein VdSCP41 to modulate immunity. Elife, 7, e34902.

Reddy, V.S., Ali, G.S. and Reddy, A.S. (2002) Genes encoding calmodulin-binding proteins in the Arabidopsis genome. J Biol Chem, 277, 9840-9852.

Sun, T., Busta, L., Zhang, Q., Ding, P., Jetter, R. and Zhang, Y. (2018) TGACG-BINDING FACTOR 1 (TGA1) and TGA4 regulate salicylic acid and pipecolic acid biosynthesis by modulating the expression of SYSTEMIC ACQUIRED RESISTANCE DEFICIENT 1 (SARD1) and CALMODULIN-BINDING PROTEIN 60g (CBP60g). The New phytologist, 217, 344-354.

Sun, T., Huang, J., Xu, Y., Verma, V., Jing, B., Sun, Y., Ruiz Orduna, A., Tian, H., Huang, X., Xia, S., Schafer, L., Jetter, R., Zhang, Y. and Li, X. (2020) Redundant CAMTA Transcription Factors Negatively Regulate the Biosynthesis of Salicylic Acid and N-Hydroxypipecolic Acid by Modulating the Expression of SARD1 and CBP60g. Mol Plant, 13, 144-156.

Sun, T. and Zhang, Y. (2021) Short-and long-distance signaling in plant defense. The Plant Journal, 105, 505-517.

Sun, T., Zhang, Y., Li, Y., Zhang, Q., Ding, Y. and Zhang, Y. (2015) ChIP-seq reveals broad roles of SARD1 and CBP60g in regulating plant immunity. Nature communications, 6, 10159.

Tian, H., Chen, S., Wu, Z., Ao, K., Yaghmaiean, H., Sun, T., Huang, W., Xu, F., Zhang, Y. and Wang, S. (2020) Activation of TIR signaling is required for pattern-triggered immunity. bioRxiv.

Truman, W., Sreekanta, S., Lu, Y., Bethke, G., Tsuda, K., Katagiri, F. and Glazebrook, J. (2013) The CALMODULIN-BINDING PROTEIN60 family includes both negative and positive regulators of plant immunity. Plant physiology, 163, 1741-1751. 
van Wersch, R., Li, X. and Zhang, Y. (2016) Mighty dwarfs: Arabidopsis autoimmune mutants and their usages in genetic dissection of plant immunity. Frontiers in plant science, $\mathbf{7}$, 1717.

van Wersch, S., Tian, L., Hoy, R. and Li, X. (2020) Plant NLRs: the whistleblowers of plant immunity. Plant Communications, 1, 100016.

Wang, L., Tsuda, K., Sato, M., Cohen, J.D., Katagiri, F. and Glazebrook, J. (2009) Arabidopsis CaM binding protein CBP60g contributes to MAMP-induced SA accumulation and is involved in disease resistance against Pseudomonas syringae. PLoS Pathog, 5, e1000301.

Wang, L., Tsuda, K., Truman, W., Sato, M., Nguyen le, V., Katagiri, F. and Glazebrook, J. (2011) CBP60g and SARD1 play partially redundant critical roles in salicylic acid signaling. Plant J, 67, 1029-1041.

Xing, H.-L., Dong, L., Wang, Z.-P., Zhang, H.-Y., Han, C.-Y., Liu, B., Wang, X.-C. and Chen, Q.-J. (2014) A CRISPR/Cas9 toolkit for multiplex genome editing in plants. BMC plant biology, $14,1-12$.

Zhang, Y., Xu, S., Ding, P., Wang, D., Cheng, Y.T., He, J., Gao, M., Xu, F., Li, Y., Zhu, Z., Li, X. and Zhang, Y. (2010a) Control of salicylic acid synthesis and systemic acquired resistance by two members of a plant-specific family of transcription factors. Proc Natl Acad Sci U S A, 107, 18220-18225.

Zhang, Y., Yang, Y., Fang, B., Gannon, P., Ding, P. and Li, X. (2010b) Arabidopsis snc2-1D activates receptor-like protein-mediated immunity transduced through WRKY70. Plant Cell, 22, 3153-3163.

Zhou, J.M. and Zhang, Y. (2020) Plant Immunity: Danger Perception and Signaling. Cell, 181, 978-989. 